\title{
Índices baseados no número de clientes para localização de itens em armazéns
}

\author{
Marcele Elisa Fontana ${ }^{a *}$, Cristiano Alexandre Virgínio Cavalcante ${ }^{b}$ \\ a*marcelelisa@gmail.com, UFPE, Brasil \\ ${ }^{\mathrm{b}}$ cristiano@ufpe.br, UFPE, Brasil
}

\begin{abstract}
Resumo
Para se obter eficiência nas operações de armazenagem, um fator importante é estabelecer a política ótima de atribuição da localização dos itens em armazéns. Para tanto, um dos índices mais difundidos e utilizados é o cuber-per-order (CO1). No entanto, observa-se que há situações em que o uso do c01 pode concentrar vantagens para uma minoria de clientes. Nesse sentido, este artigo propõe verificar as consequências da utilização de índices alternativos ( $\mathrm{COIC} \mathrm{e} \mathrm{ClC}$ ), baseados no número de clientes que demandam cada tipo de produto (item), sobre o desempenho de um armazém em relação à utilização do COl. Conclui-se que considerar o número de clientes na determinação da política ótima de atribuição de locais aos itens armazenados proporciona uma maior vantagem competitiva, uma vez que um número maior de clientes pode ser beneficiado. 1sso representa uma oportunidade de elevar o nível de serviço oferecido pelo armazém aos clientes.
\end{abstract}

Palavras-chave

Armazenamento. Serviço ao cliente. Formação de classe e localização.

\section{Introdução}

Nos últimos anos, o serviço logístico criou conveniência para os clientes, em que o não atendimento do mercado representa perda de vendas (ROBERTS; LILIEN, 1993 apud GOLDSTEIN; ALMEIDA, 2000). Nesse sentido, os armazéns mudaram seu papel estratégico para atingir as metas logísticas de menores tempos de ciclo do pedido, menor nível de inventário, menores custos e melhor serviço para o cliente, como relatam Coyle et al. (1996) apud Hsu, Chen e Chen (2005).

Um planejamento apropriado dos estoques ajuda a efetuar movimentação e armazenagem mais eficientes, o que, no final, resulta em despesas operacionais menores. Assim, de acordo com Liu (1999), um problema de localização de estoque em armazéns objetiva maximizar o espaço disponível e minimizar o total da distância percorrida ou o tempo de trajeto em todo o armazém para atender às ordens dos clientes. 0 termo inglês utilizado para definir o processo pelo qual os montantes adequados dos produtos são obtidos a partir de um local específico no estoque para cumprir as ordens (encomendas) dos clientes é denominado order picking (JANE; LAlH, 2005).

Com o objetivo de estabelecer a melhor política de localização de itens em armazéns, na literatura é encontrado o índice cube-per-order (COI), que estabelece uma ordenação dos itens para então eles serem alocados em determinados locais dentro do armazém. Esse índice relaciona as características de dimensões (cube) e a demanda (order) de cada item. No entanto, Muppani e Adil (2008a, p. 612) afirmam que "[...] uma formação de classes sob a base do índice COl pode ser subótima".

Mediante essa afirmação, verificou-se que o COI não leva em consideração o número de clientes que demandam cada produto. Por esse motivo sugere-se a utilização do número de clientes na atribuição de locais aos itens em armazém com o objetivo de minimizar as distâncias percorridas para completar as ordens dos clientes (order picking). Os índices de 
atribuição sugeridos são: o cube-per-consumer (CIC), que é a razão entre o espaço requerido e o número de clientes; e o cube-per-order and consumer (COIC), que é a razão do espaço requerido pela demanda multiplicada pelo número de clientes.

Contudo, este artigo parte da hipótese de que não considerar o número de clientes na localização dos itens em armazéns pode ter como possível consequência, dentre outras, o oferecimento de níveis de serviços mais elevados a poucos clientes em detrimento da maioria. Portanto este artigo tem por objetivo avaliar os impactos operacionais na armazenagem, mas principalmente sobre os custos de armazenagem, quando utilizado o número de clientes para a construção de um índice que é utilizando na atribuição de locais às classes de produtos armazenados. Além disso, busca-se verificar possíveis melhorias no nível de serviço oferecido aos clientes pelo armazém. Dessa forma, faz-se uma investigação de cenários simulados e gerados aleatoriamente a fim de observar o impacto do uso de índices alternativos (COIC e ClC), em comparação com o $\mathrm{COl}$, nos principais indicativos de desempenho de um armazém.

0 trabalho está organizado da seguinte forma: a seção 2 traz alguns conceitos gerais e a caracterização do problema estudado, bem como define como se chega às variáveis utilizadas na comparação do desempenho dos índices de atribuição. A seção 3 apresenta a análise dos índices para localização de itens em armazéns. Na seção 4 é realizada a simulação para verificar a aplicabilidade dos índices propostos. Na sequência é apresentada uma análise dos resultados da simulação e, por fim, algumas considerações finais são feitas na seção 6 .

\section{A atribuição da localização dos itens em armazéns}

Dada a situação concorrencial do mercado, muitas organizações procuram aumentar a satisfação do cliente como um meio de obter vantagem competitiva (BURNS; BOWLING, 2010). Davis, Chase e Aquilano (2003) definiram a satisfação do cliente como relacionada à comparação entre sua expectativa quanto ao desempenho de um serviço e a percepção desse desempenho.

Além disso, em função da política de estoques reduzidos, os clientes procuram fazer pedidos cada vez menores e com maior frequência, forçando o estoque para trás na cadeia de suprimentos. A redução do tamanho do pedido aumenta a demanda pelas operações de picking, além de dificultá-las quando se trabalha com pedidos de caixas quebradas. Somam-se a isso a variação no tamanho das embalagens com que os produtos são comercializados no varejo, aumentando o número de itens a serem controlados, processados e manuseados nos armazéns e implicando em diminuição da produtividade, maior necessidade de espaço e maiores custos administrativos (RIBEIRO; SILVA; BENVENUTO, 2006).

Assim, os clientes exigem cada vez maiores níveis de serviço, que incluem: entregas com maior frequência e consistentes, entregas no tempo certo programado, transações sem erro, além de disponibilidade de estoque (HONG, 1999). No entanto, atender a um pedido de forma precisa é desafiador, mas oferece também uma grande oportunidade para reduzir custos e melhorar, simultaneamente, o serviço para o cliente (KALAKOTA; ROBINSON, 2002). Uma empresa de sucesso, portanto, é aquela que consegue fornecer os produtos certos para os clientes certos, na hora certa e pelo preço certo (JIANG; SHANG; LIU, 2010).

0 tempo gasto em atividades de armazenagem é um fator importante para o tempo total gasto no ciclo de pedido, sendo essencial estudar meios viáveis e sustentáveis que minimizem esse tempo. Segundo Li, Chen e Liu (2008), as decisões sobre o estoque em armazenagem influenciam quase todos os principais indicadores de desempenho do armazém, tais como tempo e custo de order picking. Para Chen et al. (2005), uma vez que as atividades de armazenamento são frequentes, até mesmo uma pequena melhoria pode conseguir saldos significativos.

Contudo, os gestores estão cada vez mais interessados em encontrar a maneira mais econômica de order picking, minimizando o custo com a redução das distâncias percorridas. De acordo com Koster, Le-Duc e Roodbergen (2007), a ligação crucial entre order picking e nível de serviço é que quanto mais rápido uma ordem pode ser recuperada, mais cedo estará disponível para o envio ao cliente.

Voltando as atenções para o sistema de localização de itens em armazéns, pode-se pensar em três principais categorias, apresentadas por Hausman et al. (1976) apud Li, Chen e Liu (2008), que são: estocagem dedicada ou fixa; estocagem variável ou aleatória e estocagem classe-base. A política de armazenamento dedicada prescreve uma localização específica onde cada unidade estocada (SKU) deve ser armazenada (ROUWENHORST et al., 2000), e não se pode guardar nenhum outro item ali, mesmo que o espaço esteja vazio.

Sistemas com localização aleatória são utilizados para otimizar a área destinada ao armazenamento, uma vez que os materiais são colocados nos espaços livres existentes. Contudo, tais sistemas de localização exigem um maior controle sobre as informações relativas ao posicionamento, bem como podem aumentar os 
custos de order picking, já que cada produto pode encontrar-se em diferentes pontos do armazém. As localizações aleatória e dedicada são casos extremos de classe-base, na qual o primeiro considera uma única classe e o segundo considera uma classe para cada tipo de item (MUPPANI; ADIL, 2008a).

Para a formação das classes, Hesket (1963) apud Brynzér e Johansson (1996), propôs o índice cube-per-ordem (COI), que é definido como a relação entre o espaço requerido para o armazenamento do(s) item(s) (por unidade de armazém) e sua popularidade (demanda/período), conforme a Equação 1.

$$
\mathrm{COI}_{\mathrm{p}}=\frac{f_{p}^{*}\left[\mathrm{Max}_{\mathrm{p}}^{\mathrm{t}}\right]}{\mathrm{D}_{\mathrm{p}}}
$$

em que:

- $f_{p}$ - densidade (área necessária para estocar uma unidade de carga do produto $p$ );

- $l_{p}^{t}$ - quantidade de unidades planejadas para o armazenamento do produto $p$ durante o período $t$;

- $D_{p}$ - número total de viagens até o produto $p$ no período programado (em unidade de carga).

De acordo com Jane e Laih (2005), na regra do COl os itens são ordenados do menor para o maior valor do índice e, em seguida, os itens de menor COl são atribuídos aos locais mais próximos dos pontos 1/0 (entrada/saída), seguindo a ordenação até o final do armazém. Dessa maneira é possível minimizar as distâncias médias percorridas até cada produto e, consequentemente, reduzir o custo com a order picking.

Observou-se que o $\mathrm{COl}$ não leva em consideração o número de clientes que demandam cada item. Pensando em nível de serviço, isso pode levar a empresa a oferecer os menores tempos de concretização das ordens àqueles que demandam maior quantidade de produtos e não necessariamente ao maior número de clientes.

Devido a esse fato, propõe-se a comparação do COI com os índices COIC e CIC para determinar a melhor política de alocação de produtos em armazém, a qual eleve o nível de serviço oferecido. 0 COIC agrega à fórmula do COI o número de clientes por produto, como apresenta a Equação 2.

$\operatorname{COIC}_{\mathrm{p}}=\frac{f_{p}{ }^{*}\left[\mathrm{Max} \mathrm{I}_{\mathrm{p}}^{\mathrm{t}}\right]}{\mathrm{D}_{\mathrm{p}}{ }^{*} \mathrm{C}_{\mathrm{p}}}$

em que:

- $C_{p}$ é número de clientes que demandam o produto $p$. No $\mathrm{ClC}$, retirou-se a quantidade demandada da fórmula, sendo determinada a localização pela razão entre o espaço requerido e o número de clientes, como a Equação 3.

$\mathrm{ClC}_{\mathrm{p}}=\frac{f_{d} *\left[\mathrm{Max} \mathrm{I}_{\mathrm{p}}^{\mathrm{t}}\right]}{\mathrm{C}_{\mathrm{p}}}$

Contudo, diferentes estratégias de armazenamento podem ser utilizadas. A implementação de cada estratégia de armazenamento é uma questão operacional. A seleção de um método de order picking é uma decisão estratégica, uma vez que tem um amplo impacto em muitas outras decisões no projeto e na operacionalização do armazém (GU; GOETSCHALCKX; McGINNIS, 2007).

Para Muppani e Adil (2008a, b), a maioria dos modelos de atribuição de locais em armazéns enfatiza a relação tempo de armazenagem e order picking, mas alguns poucos trabalhos na literatura consideram a redução de espaço de armazenagem, bem como os custos relativos ao espaço e order picking na formação de classes. Este artigo utilizará a modelagem proposta por esses autores.

Esse modelo de formação de classe e localização consiste em: dado um produto $P$, sua demanda média $D_{p}$ e seu nível de inventário $l_{p}$ planejado para $T$ períodos, e o layout da área de armazenamento dividido em treliças, estabelecer as classes de produtos e alocá-los nos locais de armazenamento de modo que o custo total de armazenamento seja minimizado (custo order picking + custo espaço), explorando a redução da área de estocagem. As operações de order picking são realizadas em comando único, ou seja, é obtida apenas uma unidade de qualquer produto durante uma order picking. Dessa forma, os elementos considerados no modelo são:

Minimize

$$
\begin{aligned}
& Z=f^{*} \sum_{c} \sum_{l}\left(a_{l} \cdot y_{l c}\right) \\
& +2 h \cdot \sum_{c} *\left[\left\{\frac{\sum_{l}\left(a_{l} \cdot d_{l} \cdot y_{l c}\right)}{\sum_{l}\left(a_{l} \cdot y_{l c}\right)}\right\} * \sum_{p} D_{p}{ }^{*} x_{p c}\right]
\end{aligned}
$$

Sujeito a:

$$
I N D_{p}{ }^{*} x_{p c} \leq I N D_{p^{\prime}}{ }^{*} x_{p^{\prime} c^{\prime}} \quad \forall p \neq p^{\prime} \text { e } c<c^{\prime}
$$

$$
l^{*} y_{l c} \leq l^{* *} y_{l^{\prime} c^{\prime}} \quad \forall l \neq l \text {, e } c<c^{\prime}
$$

$$
\operatorname{Max}_{t}\left[\sum_{p} I_{p}^{t} * f_{p}^{*} x_{p c}\right] \leq \sum_{l}\left(a_{l} * y_{l c}\right) \quad \forall c
$$


$\sum_{c} x_{p c}=1$

$\forall p$

$\sum_{c} y_{l c} \leq 1$

$\forall I$

$x_{p c}, y_{l c} \in\{1,0\}$

$\forall p, c, l$.

em que:

- $\mathrm{c}-(\mathrm{c}=1,2,3, \ldots, \mathrm{C}=\mathrm{P})$ para classes;

- $l$ - $(\mathrm{l}=1,2,3, \ldots, \mathrm{L})$ para o local de armazenagem;

- $p-(p=1,2,3, \ldots$, P) para os produtos/itens;

- $t-(\mathrm{t}=1,2,3, \ldots, \mathrm{T})$ para o período de tempo;

- $I N D_{p}$ - índice utilizado para a ordenação do produto $p$;

- $a_{l}$ - área do local $l$ (em metros quadrados - $\mathrm{m}^{2}$ );

- $d_{l}$ - distância até o local $l$ a partir do ponto 1/0 (em metros - m);

- $f$ - custo do espaço no horizonte de planejamento $\left(\mathrm{R} \$ / \mathrm{m}^{2}\right)$;

- $h$ - custo de order-picking por metro percorrido $(\mathrm{R} \$ / \mathrm{m})$.

- Variáveis de decisão:

- $x_{p c}=1$, se o produto $p$ é atribuído à classe $c$;

- $x_{p c}=0$, otherwise;

- $y_{l c}=1$, se o local $l$ é atribuído à classe $c$;

- $y_{l c}=0$, otherwise.

A função objetivo (4) minimiza o custo do espaço de armazenamento e de order picking sobre o horizonte de planejamento. As restrições (5) e (6) juntas presumem que se um produto tem menor $I N D$ é atribuído à classe $c$, e o produto com maior $I N D$ é atribuído à classe c', então $c$ é alocado mais próximo ao ponto $1 / 0$ que a classe c'; (7) garante que existe espaço suficiente para armazenar o item; (8) garante que cada item seja atribuído exatamente a uma classe; (9) garante que um local seja atribuído apenas a uma classe; (10) impõe restrições binárias às variáveis de decisão.

Em suma, esse modelo de programação não linear testa todas as possíveis formações de classes dos produtos armazenados e busca aquela que minimiza os custos de armazenagem levando em consideração as características não só do armazém, mas também dos produtos armazenados, que são fundamentalmente 0 requerimento de espaço e a distância percorrida em order picking. Portanto, esse modelo é usado neste artigo, alternando-se o índice de formação das classes (IND) e verificando as consequências no armazém.

\section{Análise dos índices para localização de itens em armazéns}

A análise dos índices para localização de itens em armazéns é feita com base no modelo proposto por Muppani e Adil (2008a, b), no qual é utilizada aquela alocação dos itens que resultar no menor custo total de armazenagem. A partir dessa localização ótima devem-se verificar as porcentagens de clientes priorizados com um atendimento mais rápido, na alocação dos itens resultante, e a do índice de atribuição relatado anteriormente.

A proposta deste artigo concentra-se, portanto, no serviço prestado ao cliente. Por isso, nas simulações realizadas os clientes são divididos em grupos. Os grupos são caracterizados pelo tipo de produto que demandam, ou seja, cada cliente de um determinado grupo demandará apenas um determinado tipo de produto armazenado. Apesar de não realístico, pois na prática os clientes, em geral, demandam vários tipos de produtos, o objetivo dessa simulação é apenas servir como cenário para realizar a comparação do desempenho dos índices citados ( $\mathrm{COl}, \mathrm{COIC}$ e $\mathrm{ClC}$ ), a fim de verificar o serviço prestado ao cliente.

0 serviço prestado será medido em função do tempo de concretização das ordens. 0 tempo total de atendimento a um cliente $(k=\{1,2,3, \ldots K\})$ é o somatório do tempo gasto para a order picking de todos os produtos da sua ordem, conforme a Equação 11.

$$
\text { Temp_ } C_{k}=\sum_{k=1}^{K} \frac{\text { dist }_{p}}{v} \quad \forall p, k
$$

em que:

- $v$ - é a velocidade do equipamento de order picking;

- dist $_{p}$ - é a distância média até o produto $p$, que é a mesma até o local $l(d)$ onde o produto está alocado.

A partir desse ponto calcula-se, então, a denominada "porcentagem de priorização de clientes (\%)”, ou seja, a quantidade de clientes, em percentual, que se beneficiará do menor tempo de atendimento de suas ordens quando comparados os resultados de cada índice de atribuição.

Neste artigo é suposto que o cliente ou grupo de clientes demanda apenas um tipo de produto e vice-versa. Portanto, o procedimento para calcular a porcentagem de priorização de clientes é o seguinte: para cada tipo de produto armazenado é verificado qual dos índices de atribuição (CIC, $\mathrm{COI}$ ou $\mathrm{COIC}$ ) resultou em uma localização que forneça o menor tempo de concretização das ordens dos clientes naquele item. Isso é verificado para todos os produtos armazenados. 
Em seguida, para cada índice utilizado é somado o número de clientes naqueles produtos em que o indice ofereceu o menor tempo de atendimento ou concretização das ordens. Por fim, a porcentagem de priorização de clientes em cada índice será a relação entre o número total de clientes beneficiados pelo indice e o número total de clientes analisados. Para exemplificar, suponha que de um total de 200 clientes o menor tempo de concretização das ordens se deu da seguinte maneira: 40 clientes pelo $\mathrm{COI}, 70$ pelo $\mathrm{COIC}$ e 90 pelo CIC. Dessa maneira, têm-se as seguintes porcentagens de priorização: 20\% pelo COI, 35\% pelo COIC e 45\% pelo ClC.

Observa-se que, nesse caso, os clientes são igualmente importantes. Sendo assim, deseja-se beneficiar o maior número destes. Essa poderia ser a situação em que se quer definir o nível de serviço básico, ou seja, o nível de serviço mínimo ofertado para todos os clientes de forma indiscriminada.

Destaque-se que esse nível de serviço, quando distante do praticado pela concorrência, coloca a empresa fora do cenário competitivo, sendo de extrema importância a definição coerente do serviço básico (BOWERSOX; CLOSS, 2009). Por outro lado, outra proposta pode ser ponderar os índices com a importância relativa do cliente, quando são identificadas as diferentes classes de clientes. A Figura 1 resume os procedimentos realizados neste artigo.

Em suma, neste trabalho as variáveis descritas nesta secção serão utilizadas para comparar o desempenho de cada índice de atribuição. Contudo, a maior contribuição será verificar o serviço oferecido pelo armazém às operações realizadas, a partir da atribuição resultante de cada índice, por meio do cálculo das porcentagens de priorização dos clientes. É importante destacar que a política ótima de atribuição em cada cenário analisado e por cada índice utilizado se baseia no modelo de formação de classe e localização proposto por Muppani e Adil (2008a, b).

\section{Modelo de simulação}

Assume-se que cada local de estocagem é uniformemente utilizado, os pontos atribuídos são distribuídos de forma homogênea no espaço alocado

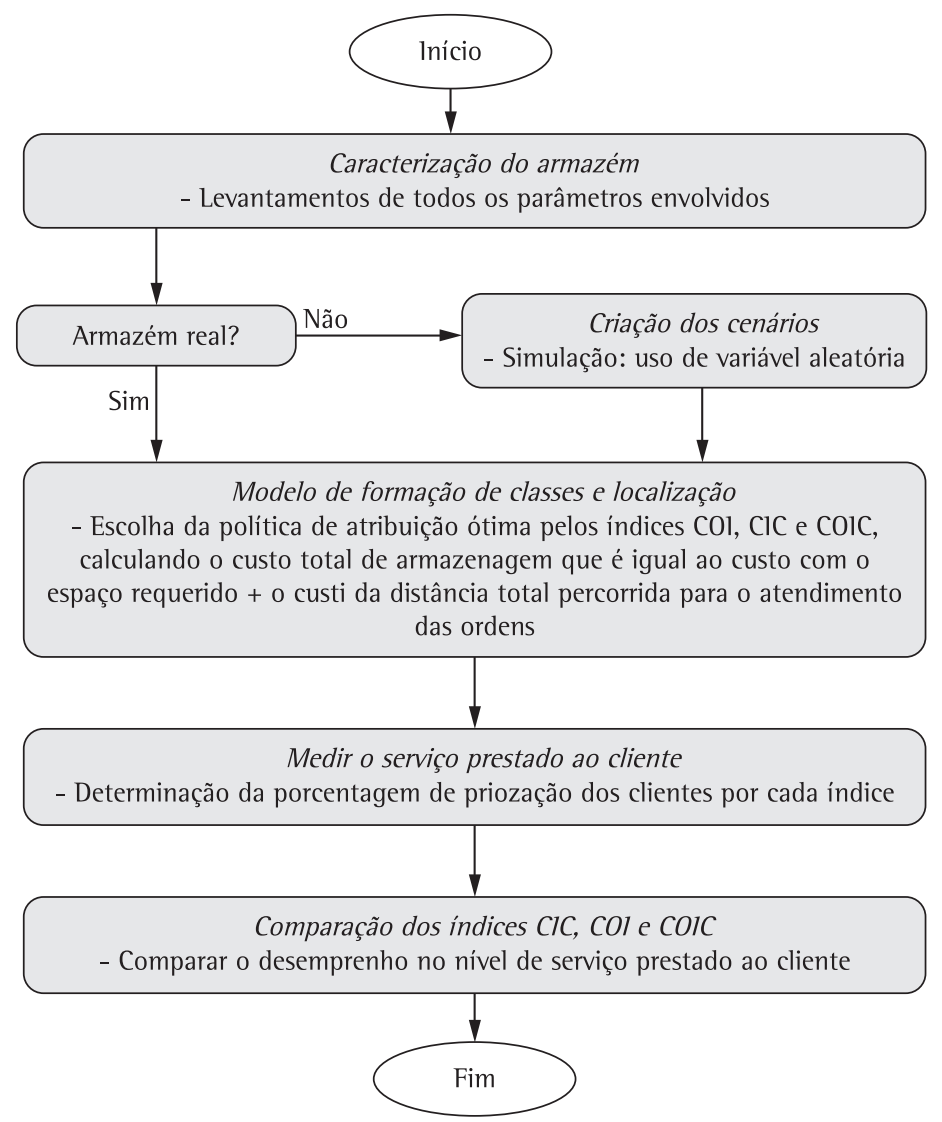

Figura 1. Resumo dos procedimentos adotados no artigo. 
para a classe e todos os itens são armazenados e transportados em suportes idênticos. 0 armazém simulado é retangular, dividido em células (treliças) de $1,0 \times 1,0 \mathrm{~m}$, conforme a Figura 2, adaptada de Chew e Tang (1999). 0 layout utilizado no armazém considera 10 colunas (sentido $x$ da Figura 1). Estabeleceu-se que todos os produtos são empilhados em no máximo 10 niveis (pilhas).

Foram considerados quatro períodos de análise, em que cada um representa uma semana $(t=4)$, totalizando um mês. Presume-se que oscilações de demanda e inventário ocorrem durante o mês, não havendo variações significativas no decorrer do ano. Na simulação são armazenados apenas 10 produtos distintos.

Dez cenários foram gerados ao acaso por meio de uma variável aleatória que oscila entre [0, 1]. A aleatoriedade é interessante para evitar vieses nos resultados. Estabeleceu-se uma faixa de 10 a 1.500 unidades semanais para a quantidade demandada de cada produto e uma faixa de 1 a 55 para o número de clientes que demanda cada produto. A demanda utilizada nos cálculos é a média dos quatro períodos, respeitando-se a restrição de ser maior ou igual ao número de clientes que demanda o produto.

Para exemplificar, suponha que a variável aleatória da demanda no período $t$, para o produto $p_{1}$ é igual a 0,35 . Dessa maneira, a demanda $d$, será igual a: $d_{1}=1500 * 0,35=525$ unidades. Essa variável aleatória é gerada para todos os produtos quatro vezes, que representarão os quatro períodos constituintes

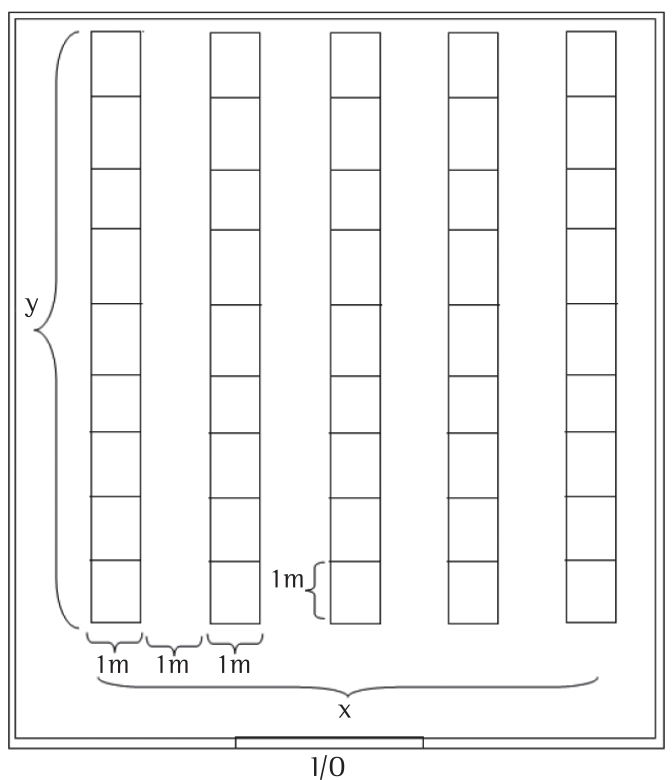

Figura 2. llustração do layout da área de estocagem do armazém. de um cenário. E este procedimento é repetido dez vezes, que serão os dez cenários simulados. 0 mesmo procedimento é aplicado para o número de clientes. Contudo, o número de clientes será o mesmo nos quatro períodos, apenas alterando-se entre os dez cenários. Além disso, para garantir que a demanda não seja nula foi estabelecido um valor mínimo de 10 unidades. Assim, caso essa demanda esteja abaixo de 10 unidades, o valor é eliminado e um novo valor para aquele período é sorteado aleatoriamente; caso este seja maior do que 10, o valor será utilizado.

0 inventário, ou seja, a quantidade de itens em estoque no período t é a relação entre a demanda média do produto (calculada como descrito anteriormente) e uma variável aleatória $[0,1]$ que simulará as variações na quantidade de cada produto estocada em cada período $t$. Os valores gerados para a quantidade demandada, número de clientes e unidades em inventário devem ser arredondados para o inteiro mais próximo no sentido do $+\infty$, os quais representam os valores reais utilizados, pela impossibilidade de existirem valores decimais. A densidade é exatamente o valor da variável aleatória, ou seja, a densidade dos produtos encontra-se na faixa entre 0 e $1 \mathrm{~m}^{2} / \mathrm{un}$, devendo sempre ser diferente de zero.

0 espaço de armazenagem requerido relaciona-se com o inventário. Assim, o espaço utilizado é a quantidade de unidades em estoque por sua densidade, dividida pelos 10 níveis possíveis para cada produto a ser empilhado. Pode-se perceber, então, que há uma oscilação do espaço requerido nos quatro períodos. Uma economia de espaço é possível quando se agregam produtos que apresentam oscilações na utilização de espaço, para mais e para menos, em períodos diferentes.

Para visualizar essa economia de espaço, suponha o requerimento de espaço em dois períodos distintos, definidos como $1=\left\{1_{1}, 1_{2}\right\}$. Sejam considerados dois produtos com os seguintes requerimentos de espaço, em $m^{2}: A=\{10,5\}$ e $B=\{10,15\}$. Em uma armazenagem dedicada, ou seja, existe um espaço dedicado no armazém para cada item individualmente, o espaço total necessário para alocar A é de $10 \mathrm{~m}^{2} \mathrm{e}$ o espaço necessário para alocar B é de $15 \mathrm{~m}^{2}$. Esses são os maiores requerimentos de espaço por cada item (considerando-se apenas dois períodos no exemplo). Assim, para garantir que haverá disponibilidade de espaço em todos os t períodos seria necessário um espaço total de $25 \mathrm{~m}^{2}(10+15)$.

Porém, se esses dois produtos fossem agregados em uma classe de armazenagem, o espaço total necessário em cada período seria de $l_{1}=\left\{A_{1}+B_{1}\right\}=10+10=20 \mathrm{~m}^{2}$ e $l_{2}=\left\{A_{2}+B_{2}\right\}=5+15=20 \mathrm{~m}^{2}$, ou seja, $1=\{20,20\}$. Portanto, seria necessário reservar apenas $20 \mathrm{~m}^{2}$ para a armazenagem desses dois itens. Obtém-se, assim, um 
melhor aproveitamento do espaço com a agregação desses produtos. Caso eles não sejam agregados na mesma classe de armazenagem, o espaço requerido por A continuará igual a $10 \mathrm{~m}^{2}$ e por $B$ também continuará a ser $15 \mathrm{~m}^{2}$, somando os $25 \mathrm{~m}^{2}$. Não ocorrerá, portanto, a redução do espaço desejado.

No que se refere à velocidade de order picking, considera-se que seja de um metro por segundo $(1 \mathrm{~m} / \mathrm{s})$. Assim, os dados obtidos representam tanto a distância percorrida (metros) quanto o tempo gasto (segundos). Em todos os cenários são armazenados 10 produtos com características distintas, aleatoriamente determinadas. Estabeleceu-se que todos os produtos considerados no primeiro período são também demandados nos demais.

Os valores apresentados no decorrer das simulações referem-se à média, representado apenas um período (mês). As distâncias em order picking representam a ida até o produto e a volta ao ponto de origem (1/0), considerando-se que a operação sempre inicie o processo no ponto $1 / 0$. As ordens dos clientes são atendidas pelo mecanismo PEPS (primeiro que entra - primeiro que sai).

\section{Análise dos resultados da simulação}

Com base na caracterização da simulação realizada na secção 3, busca-se estudar o comportamento dos índices de atribuição ( $\mathrm{COl}, \mathrm{ClC}$ e $\mathrm{COIC}$ ). Para determinar a melhor política de estocagem em cada índice de atribuição (ClC, $\mathrm{COl}$ e $\mathrm{COIC}$ ), foi considerado o custo de $\mathrm{R} \$ 1,50 / \mathrm{m}^{2}$ para o espaço utilizado e de $\mathrm{R} \$ 0,0025 / \mathrm{m}$ percorrido em order picking. Estes valores são baseados no trabalho de Muppani e Adil (2008a). Outros custos decorrentes da armazenagem não foram considerados.

A Tabela 1 apresenta o espaço requerido, a distância total percorrida para concretizar as ordens dos clientes e o custo total das operações de armazenagem (sendo: custo total $=$ espaço requerido + distância percorrida). Esses valores são da política ótima de atribuição de locais aos itens em armazém, ou seja, aquela que oferece o menor custo total em cada índice de atribuição utilizado (CIC, $\mathrm{COI}$ e $\mathrm{COIC}$ ) e para os dez cenários simulados aleatoriamente.

Conforme valores apresentados na Tabela 1, a menor utilização do espaço, isto é, a maior economia

Tabela 1. Espaço requerido, distância total percorrida e custo total de armazenagem nos três índices de atribuição.

\begin{tabular}{|c|c|c|c|c|}
\hline Cenário & Índice & Espaço requerido $\left(\mathrm{m}^{2}\right)$ & Distância total percorrida $(\mathrm{m})$ & Custo total $\left(\mathrm{R} \$ / \mathrm{m}^{2}+\mathrm{R} \$ / \mathrm{m}\right)$ \\
\hline \multirow{3}{*}{1} & $\mathrm{ClC}$ & 209,82 & $60.048,50$ & 614,97 \\
\hline & $\mathrm{COl}$ & 209,96 & $56.526,74$ & 597,57 \\
\hline & $\mathrm{COIC}$ & 209,82 & $60.708,50$ & 618,27 \\
\hline \multirow{3}{*}{2} & $\mathrm{ClC}$ & 128,66 & $19.047,27$ & 288,23 \\
\hline & $\mathrm{COl}$ & 130,41 & $20.937,77$ & 300,30 \\
\hline & $\mathrm{COIC}$ & 128,45 & $21.524,81$ & 300,30 \\
\hline \multirow{3}{*}{3} & $\mathrm{ClC}$ & 191,30 & $28.196,02$ & 427,93 \\
\hline & $\mathrm{CO1}$ & 191,16 & $28.594,50$ & 429,71 \\
\hline & $\mathrm{COIC}$ & 191,16 & $28.594,50$ & 429,71 \\
\hline \multirow{3}{*}{4} & $\mathrm{ClC}$ & 127,02 & $21.316,18$ & 297,11 \\
\hline & $\mathrm{CO}$ & 123,67 & $19.580,41$ & 283,41 \\
\hline & $\mathrm{COIC}$ & 128,89 & $21.047,95$ & 298,58 \\
\hline \multirow{3}{*}{5} & $\mathrm{ClC}$ & 194,09 & $46.578,44$ & 524,03 \\
\hline & $\mathrm{COl}$ & 189,21 & $39.855,93$ & 483,09 \\
\hline & $\mathrm{COIC}$ & 186,18 & $50.492,95$ & 531,74 \\
\hline \multirow{3}{*}{6} & $\mathrm{ClC}$ & 160,05 & $31.017,10$ & 395,16 \\
\hline & $\mathrm{CO}$ & 163,49 & $25.713,99$ & 373,80 \\
\hline & $\mathrm{COIC}$ & 176,36 & $28.121,72$ & 405,16 \\
\hline \multirow{3}{*}{7} & $\mathrm{ClC}$ & 131,25 & $26.776,36$ & 330,76 \\
\hline & $\mathrm{COl}$ & 131,25 & $26.776,36$ & 330,76 \\
\hline & $\mathrm{COIC}$ & 131,60 & $26.455,48$ & 329,68 \\
\hline \multirow{3}{*}{8} & $\mathrm{ClC}$ & 109,14 & $24.529,00$ & 286,35 \\
\hline & $\mathrm{COl}$ & 109,86 & $19.640,17$ & 262,98 \\
\hline & $\mathrm{COIC}$ & 112,14 & $24.412,90$ & 290,28 \\
\hline \multirow{3}{*}{9} & $\mathrm{CIC}$ & 135,68 & $21.773,06$ & 312,38 \\
\hline & $\mathrm{COl}$ & 136,33 & $19.413,46$ & 301,56 \\
\hline & $\mathrm{COIC}$ & 135,68 & $18.261,38$ & 294,82 \\
\hline \multirow{3}{*}{10} & $\mathrm{CIC}$ & 176,88 & $39.978,50$ & 465,22 \\
\hline & $\mathrm{CO} 1$ & 181,93 & $36.707,59$ & 456,44 \\
\hline & $\mathrm{COIC}$ & 179,01 & $39.555,85$ & 466,29 \\
\hline
\end{tabular}


Tabela 2. Porcentagem de clientes priorizados (\%).

\begin{tabular}{ccccc}
\hline Cenários & $\begin{array}{c}\text { Número de } \\
\text { clientes }\end{array}$ & CIC $(\%)$ & COI (\%) & COIC (\%) \\
\hline 1 & 222 & 81,08 & 38,74 & 77,93 \\
2 & 195 & 47,18 & 53,33 & 38,97 \\
3 & 169 & 77,51 & 63,31 & 63,31 \\
4 & 221 & 57,47 & 4,98 & 37,56 \\
5 & 203 & 15,76 & 30,54 & 53,69 \\
6 & 147 & 82,99 & 1,36 & 53,74 \\
7 & 95 & 18,95 & 18,95 & 98,95 \\
8 & 174 & 86,21 & 12,64 & 24,14 \\
9 & 179 & 37,99 & 45,25 & 46,37 \\
10 & 102 & 60,78 & 1,96 & 70,59 \\
\hline
\end{tabular}

de espaço do armazém ocorreu em 50\% dos cenários pelo $\mathrm{ClC}$ e COIC, e em 30\% pelo COI; enquanto a menor distância total percorrida foi atingida pelo CIC e COIC em 20\% dos cenários, e em $60 \%$ deles pelo COl. Da mesma forma, o custo total foi menor em 60\% dos cenários pelo COl e em 20\% deles pelo $\mathrm{CIC}$ e COIC.

Contudo, o que se utiliza desses resultados é a alocação ótima resultante de cada índice para medir o serviço oferecido aos clientes pelo armazém. Aqui, esse nível de serviço é medido pela porcentagem de priorização dos clientes, ou seja, a quantidade de clientes aos quais cada índice oferece menor tempo de atendimento total da ordem. Tais valores podem ser vistos na Tabela 2.

Em 50\% dos cenários, o $\mathrm{ClC}$ oferece menor tempo para a concretização das ordens dos clientes; em 40\% deles, é o COIC que oferece; e em apenas 10\% dos cenários esse menor tempo de concretização da ordem é oferecido pelo COl, conforme valores apontados na Tabela 2. Esses valores demonstram que ao se considerar o número de clientes que demandam cada produto na formação de classe e na localização dos itens em armazéns, há uma maior informação sobre os clientes e, assim, a alocação se torna mais eficiente em termos de rapidez no atendimento desses demandantes. Dessa forma, o armazém tem um nicho para possíveis melhorias no nível do serviço oferecido aos clientes.

\section{Considerações finais}

Este trabalho teve início com a hipótese de que não considerar o número de clientes na alocação de itens em armazéns pode ter como possível consequência, dentre outras, o oferecimento de níveis de serviços mais elevados a poucos clientes em detrimento da maioria. Verificou-se que, sendo a armazenagem uma parte importante na concretização de um pedido, vincular suas operações aos clientes, como foi o caso apresentando de atribuição de locais aos itens pelo número de clientes, a empresa disponibiliza vantagens competitivas, minimizando o tempo de concretização das ordens dos clientes. Logo, isso proporcionará uma elevação no nível de satisfação percebido pelos clientes, principalmente se eles forem sensíveis ao critério de desempenho relacionado à rapidez de atendimento.

Situação contrária é quando para a empresa os clientes têm importância relativa distinta. lsso pode correr quando o armazém tem alguns produtos de alto valor agregado e outros produtos de baixo valor ou, então, quando alguns clientes demandam a maior porcentagem dos produtos, exigindo um serviço muitas vezes personalizado. Nesse ponto, uma análise mais detalhada deve ser feita e é deixada como sugestão para trabalhos futuros. Porém fica claro que a alocação baseada no índice COI pode não ser a mais apropriada, pois não considera as características dos clientes.

Contudo, deve-se frisar que a escolha de um índice determinante da melhor política de alocação depende do cenário em que o armazém está inserido, dos custos que envolvem suas atividades e, principalmente, dos objetivos de sua gerência, como, por exemplo, se o armazém prioriza a redução do espaço utilizado, a minimização das distâncias percorridas, ou ambos. Nesse momento pode-se afirmar que a modelagem do armazém não é estática, devendo ser revista quando os objetivos e os custos do armazém forem alterados.

\section{Referências}

BOWERSOX, D. J.; ClOSS, D. J. Logística Empresarial: o processo de integração da cadeia de suprimentos. 7 . reimpr. São Paulo: Atlas, 2009. PMCid:2795220.

BRYNZÉR, H.; JOHANSSON, M.l. Storage location assignment: Using the product structure to reduce order picking times. International Journal of Production Economics, v. 46, p. 595-603, Dec. 1996. http://dx.doi. org/10.1016/0925-5273(94)00091-3

BURNS, G. N.; BOWLING, N. A. Dispositional Approach to Customer Satisfaction and Behavior. Journal of Business and Psychology, v. 25, p. 99-107, Mar. 2010. http:// dx.doi.org/10.1007/s10869-009-9129-x

CHEN, M. et al. Aggregation of orders in distribution centers using data mining. Expert Systems with Applications, v. 28, p. 453-460, Apr. 2005. http://dx.doi.org/10.1016/j. eswa.2004.12.006

CHEW, E. P.; TANG, L. C. Travel time analysis for general item location assignment in a rectangular warehouse. European Journal of Operational Research, v. 112, p. 582-597, Feb. 1999. http://dx.doi.org/10.1016/ S0377-2217(97)00416-5

DAVIS, M.; CHASE, R. B; AQUILANO, N. J. Fundamentos da administração da produção. 3. ed. Bookman, 2003. 
GOLDSTEIN, M.; ALMEIDA, H. S. Crítica dos modelos integrativos de comportamento do consumidor. Revista de Administração, v. 35, n. 1, jan./mar. 2000.

GU, J.; GOETSCHALCKX, M.; McGINNIS, L. F. Research on warehouse operation: A comprehensive review. European Journal of Operational Research, v. 177, p. 1-21, Feb. 2007. http://dx.doi.org/10.1016/j.ejor.2006.02.025

JANE, C.; LAIH, Y. A clustering algorithm for item assignment in a synchronized zone order picking system. European Journal of Operational Research, v. 155, p. 489-496, Oct. 2005. http://dx.doi.org/10.1016/j.ejor.2004.01.042

JIANG, Y. C.; SHANG, J.; LIU, Y. Z. Maximizing customer satisfaction through an online recommendation system: A novel associative classification model. Decision Support Systems, v. 48, p. 470-479, Feb. 2010. http://dx.doi. org/10.1016/j.dss.2009.06.006

HONG, Y. C. Gestão de estoques na cadeia de logística integrada - Supply chain. São Paulo: Atlas, 1999.

HSU, C.; CHEN, K.; CHEN, M. Batching orders in warehouses by minimizing travel distance with genetic algorithms. Computers in Industry, v. 56, p. 169-178, Feb. 2005. http://dx.doi.org/10.1016/j.compind.2004.06.001

KALAKOTA, R.; ROBINSON, M. E-business - estratégias para alcançar o sucesso: no mundo digital. 2. ed. Bookman, 2002. v. 1.

KOSTER, R. DE; LE-DUC, T.; ROODBERGEN, K. J. Design and control of warehouse order picking: a literature review. European Journal of Operational Research, v. 182, p. 481-501, 2007. http://dx.doi.org/10.1016/j. ejor.2006.07.009

Ll, M.; CHEN, X.; LIU, C. Pareto and niche genetic algorithm for storage location assignment optimization problem. In: INTERNATIONAL CONFERENCE ON INNOVATIVE
COMPUTING INFORMATION AND CONTROL, 3., 2008, Jhongli. Proceedings... IEE, 2008.

LIU, C.-M. Clustering techniques for stock location and orderpicking in a distribution center. Computers \& Operations Research, v. 26, p. 989-1002, Sept. 1999. http://dx.doi. org/10.1016/S0305-0548(99)00026-X

MUPPANI, V. R.; ADIL, G. K. Efficient formation of storage classes forwarehouse storage location assignment: A simulated annealing approach. OmegaInternational Journal of Management Science, v. 36, p. 609-618, Aug. 2008a. http://dx.doi.org/10.1016/j. omega.2007.01.006

MUPPANI, V. R.; ADIL, G. K. A branch and bound algorithm for class based storage location assignment. European Journal of Operational Research, v. 189, p. 492-507, Sept. 2008b. http://dx.doi.org/10.1016/j. ejor.2007.05.050

ROUWENHORST, B. et al. Warehouse design and control: Framework and literature review. European Journal of Operational Research, v. 122, p. 515-533, May 2000. http://dx.doi.org/10.1016/S0377-2217(99)00020-X

RIBEIRO, P. C. C.; SILVA, L. A. F.; BENVENUTO, S. R. S. 0 uso de tecnologia da informação em serviços de armazenagem. Produção, v. 16, n. 3, p. 526-537, set./dez. 2006. http:// dx.doi.org/10.1590/S0103-65132006000300013

\section{Agradecimentos}

Os autores agradecem ao Conselho Nacional de Desenvolvimento Científico e Tecnológico - CNPq e à Coordenação de Aperfeiçoamento de Pessoal de Nivel Superior - CAPES pelo apoio financeiro fornecido durante este estudo.

\title{
Indexes based on the number of customers to locate items in warehouse
}

\begin{abstract}
An important factor to obtain efficiency of storage operations is to establish an optimal policy for storage location assignment. To this end, one of the indexes more circulated and used is the Cuber-per-Order (COI). However, it is observed that there are situations where the use of $\mathrm{COl}$ can concentrate advantages to a minority of clients. In this sense, this paper proposes to verify the effects on the performance of a warehouse when using alternative indexes (COIC and $\mathrm{CIC}$ ), based on the number of clients that require each type of product (item), compared to the COI. It was possible to conclude that when the number of clients is considered to determine optimal policy for storage location assignment, the warehouse can acquire a competitive advantage, since the largest number of clients can be benefited. This represents an opportunity to raise the service level offered by the warehouse to its clients.
\end{abstract}

Keywords

Storage. Customer service. Class formation and location. 